\title{
Small diameter delivery system allows expandable metal biliary stent placement using a pediatric colonoscope in surgically altered anatomy
}

Endoscopic retrograde cholangiopancreatography (ERCP) is increasingly performed in patients with surgically altered anatomy [1]. Pediatric colonoscopes may be needed because of the increased flexibility but the small diameter working channel only allows placement of 7-Fr plastic stents. We report two cases of patients with surgically altered anatomy, in whom successful placement of a new self-expandable metal stent (SEMS) with a 6-Fr delivery system (Zilver, Cook Endoscopy, Winston-Salem, North Carolina, USA) allowed palliation of malignant biliary obstruction using a pediatric colonoscope.

A 79-year-old man with remote subtotal gastrostomy and RouX-en-Y reconstruction presented with obstructive jaundice due to unresectable pancreatic cancer. ERC was performed using a variable stiffness pediatric colonoscope (PCF-Q180AL, Olympus Corporation, Center Valley, Pennsylvania, USA) which was passed through an angulated afferent limb to the major papilla. Cholangiography showed a 3-cm distal bile duct stricture. A $10 \mathrm{~mm} \times 6 \mathrm{~cm}$ long SEMS was deployed across the stricture and into the duodenum ( $\bullet$ Fig. 1).

A 58-year-old man with recurrent pancreatic cancer after pancreaticoduodenectomy presented with acute cholangitis due to a hepaticojejunal anastomotic stricture. An adult colonoscope (CF-H180AL, Olympus) was passed into the afferent limb but could not be advanced to the biliary anastomosis because of severe fixation and angulation. A pediatric colonoscope (Olympus) was passed easily to the hepaticojejunal anastomosis. A guide wire was advanced into the right intrahepatic biliary tree and a $10 \mathrm{~mm} \times 4 \mathrm{~cm}$ SEMS was deployed across the hepaticojejunal anastomosis. A second $10 \mathrm{~mm} \times 6 \mathrm{~cm}$ SEMS was deployed through the interstices of the first stent and into left intrahepatic system (๑ Fig. 2).
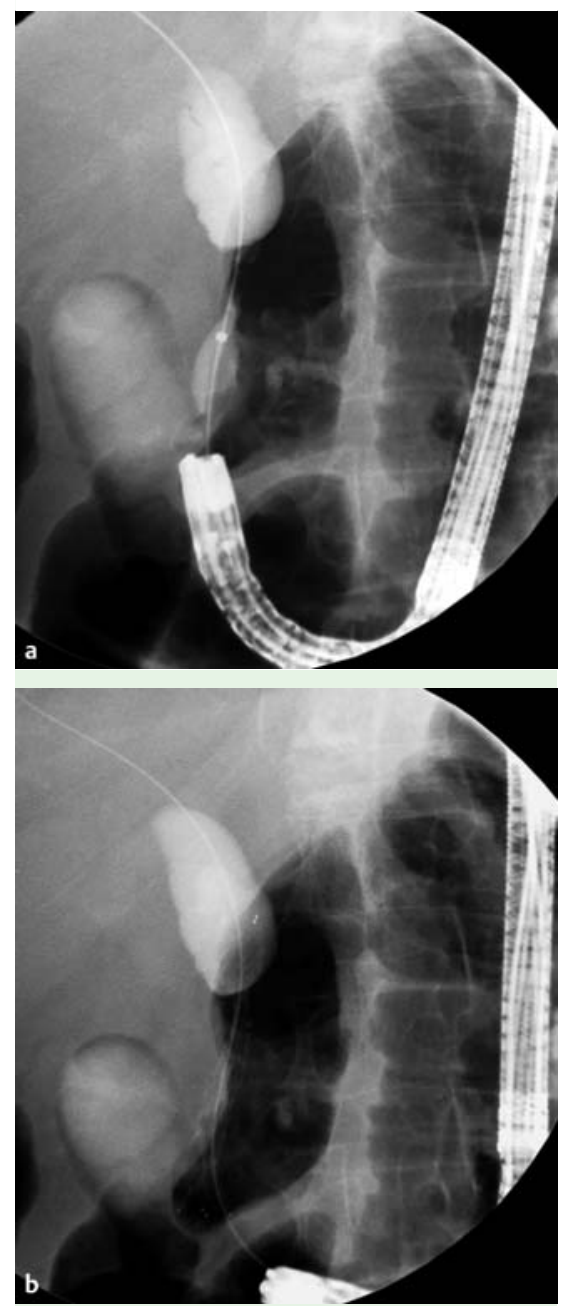

Fig. 1 SEMS placement in patient with antrectomy and Roux-en-Y gastrojejunostomy. a Cholangiogram shows malignant distal bile duct stricture. $\mathbf{b}$ Successful deployment of stent across stricture.

SEMS with small diameter delivery systems have been used to facilitate bilateral, side-by-side biliary stents to palliate malignant hilar obstruction [2]. Our two cases illustrate an additional benefit that allows placement though small working channel colonoscopes in patients with surgically altered anatomy.
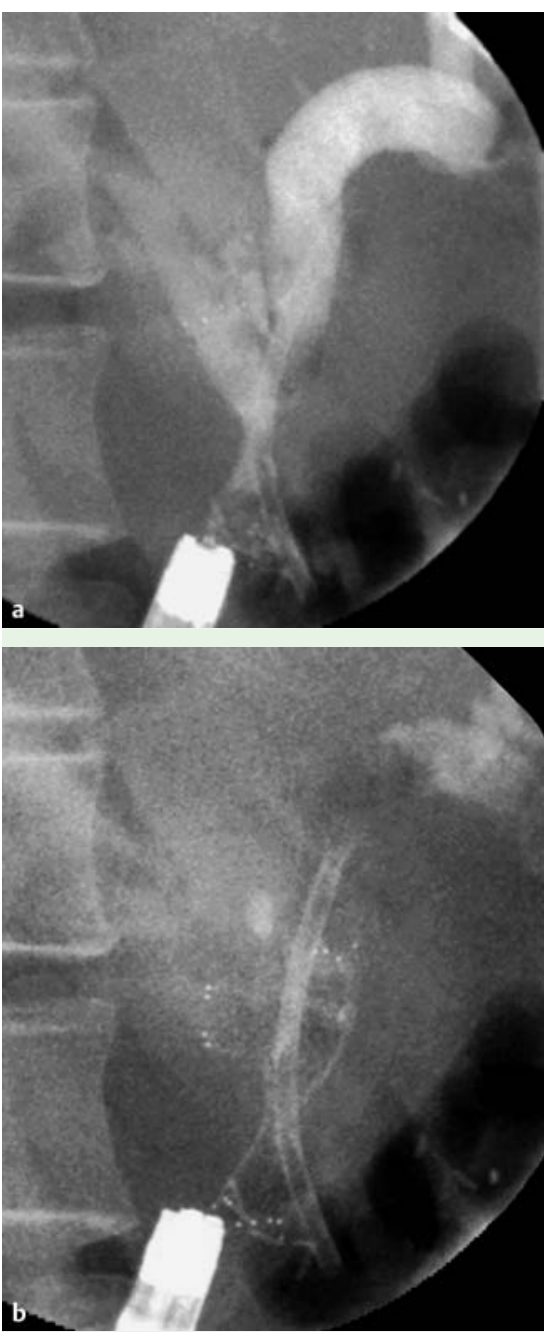

Fig. 2 Bilateral SEMS placement in patient with previous Whipple and presence of an occluded 10-Fr plastic stent placed 3 months previously. a Radiographic image after placement of right hepatic duct SEMS across hepaticojejunal stricture and injection of contrast into left system. b Successful deployment of second SEMS stent through initial SEMS and alongside occluded plastic stent.

Endoscopy_UCTN_Code_TTT_1AR_2AZ

Competing interests: None 


\section{A. Saleem, T. H. Baron}

Division of Gastroenterology and

Hepatology, Mayo Clinic, Rochester,

Minnesota, USA

\section{References}

1 Itoi T, Sofuni A, Itokawa F. Large dilating balloon to allow endoscope insertion for successful endoscopic retrograde cholangiopancreatography in patients with surgically altered anatomy (with video). J Hepatobiliary Pancreat Sci 2010; 17: 725-728
2 Chennat J, Waxman I. Initial performance profile of a new $6 \mathrm{~F}$ self-expanding metal stent for palliation of malignant hilar biliary obstruction. Gastrointest Endosc 2010; 72: 632-636

Bibliography

DOI $10.1055 / \mathrm{s}-0030-1255894$

Endoscopy 2011; 43: E69-E70

(c) Georg Thieme Verlag KG Stuttgart · New York . ISSN 0013-726X
Corresponding author

\section{T. H. Baron}

Division of Gastroenterology and Hepatology

Mayo Clinic

200 First Street SW

Charlton 8A

Rochester

MN 55905

USA

Fax: +507-266-3939

baron.todd@mayo.edu 